ISSN: 2386-3919 - e-ISSN: 2386-3927

DOI: http://dx.doi.org/10.14201/et2016341161177

\title{
EMPLEABILIDAD Y OCUPACIÓN LABORAL DE LOS EGRESADOS DEL MÁSTER EN FORMACIÓN DEL PROFESORADO DE EDUCACIÓN SECUNDARIA OBLIGATORIA, BACHILLERATO Y FORMACIÓN PROFESIONAL
}

\section{Employability and occupation of graduates of the master teacher training in compulsory secondary education and training bachelor}

María Ángeles Pascual Sevillano, Sergio Díaz Menéndez

y Celestino RodríGuEz PÉREZ

Universidad de Oviedo

Recibido: 27-07-2015; Aceptado: 03-03-2016; Publicado: 30-05-2016

BIBLID [2386-3919 (2016) 34, 1; 161-177]

Ref. Bibl. MARÍA ÁNGELES PASCUAL SEVILLANO, SERGIO DÍAZ MENÉNDEZ y CELESTINO RODRÍGUEZ PÉREZ. Empleabilidad y ocupación laboral de los egresados del Máster en Formación del Profesorado de Educación Secundaria Obligatoria, Bachillerato y Formación Profesional. Enseñanza \& Teaching, 34, 1-2016, 161-177.

RESUMEN: Se presentan los resultados del estudio llevado a cabo en la Universidad de Oviedo para conocer la empleabilidad y la mejora laboral que obtienen actualmente los egresados del Máster profesionalizante en Formación del Profesorado de Educación Secundaria Obligatoria, Bachillerato y Formación Profesional. Este estudio tiene como finalidad analizar las tendencias relacionadas con la ocupación, sueldo y tiempo que tardan en colocarse en el mercado laboral, así como la satisfacción con la formación recibida y la aplicación de los conocimientos adquiridos en el empleo obtenido. 
MARÍA ÁNGELES PASCUAL, SERGIO DÍAZ Y CELESTINO RODRÍGUEZ

EMPLEABILIDAD Y OCUPACIÓN LABORAL DE LOS EGRESADOS DEL MÁSTER EN FORMACIÓN

DEL PROFESORADO DE EDUCACIÓN SECUNDARIA OBLIGATORIA, BACHILLERATO Y FORMACIÓN...

Este tipo de estudios de egresados se ha implementado en las universidades en aras de introducir elementos de calidad en los sistemas de evaluación y como tal es contemplado en el Programa AUDIT de la Agencia Nacional de Evaluación de la Calidad y Acreditación (ANECA) para el Estado español. El estudio ha considerado una cohorte de 4 generaciones de egresados del máster de la Facultad de Formación del Profesorado y Educación. El método de trabajo utilizado fue cuantitativo de tipo descriptivo y transversal. La población objeto de estudio es de 682 alumnos. Se utilizó para ello una encuesta estructurada, diseñada «ad hoc», de 13 ítems (EnEgMfp). En el caso de la Universidad de Oviedo (UO) se ha habilitado desde la Unidad Técnica de Calidad el Procedimiento PD-SGIC-UO-1.2.4.vo7. Las conclusiones más relevantes, aunque ofrecen un panorama marcado por la coyuntura económica del país, son ciertamente esperanzadoras, un $52 \%$ de los encuestados dicen haber encontrado empleo, aunque de estos solo la mitad lo hace en el ámbito relacionado con las competencias adquiridas en el máster. La mitad de estos empleos son de carácter temporal.

Palabras clave: seguimiento de egresados; estudios de posgrado; situación laboral titulados universitarios; máster en formación del profesorado de educación secundaria.

SUMMARY: We present here the results of a study developed in the University of Oviedo to know the employability and the working improvement which are enjoying the postgraduates from the professional Master's Degree in Secondary, Languages and Vocational Training Teaching. The aim of this research is to study the tendencies related to the occupation, revenue and the time that they need to find their place in the labour market. Moreover, we would like to find out up to which extent the qualification received and the implementation of the competences acquired meet their needs in their position.

This type of study of postgraduates has been implemented at a university level in order to introduce elements of quality in assessment systems, and this is how it is conceived in the AUDIT Programme by the National Agency for the Assessment of Quality and Accreditation (ANECA) for the Spanish state. For our study a cohort of four generations of postgraduates has been considered from the master's degree in Secondary Teaching. The methodology consisted on descriptive and transversal quantitative work. The population which has constituted the object of our study comprehended 682 postgraduates. For this purpose, a structured survey has been used, designed ad hoc with 13 items (EnEgMfp). In the case of the University of Oviedo (UO) the procedure PD-SGIC-UO-1.2.4.vO7 has been authorised. The most relevant conclusions are certainly hopeful, albeit negatively influenced by the economic crisis, since $52 \%$ of the surveyed postgraduates assert to have found a post, although just half of them in the field they have qualified for with the master's degree. Half of these posts are temporary.

Key words: monitoring of graduates; postgraduate degrees; working situation; master's degree in Secondary Teaching. 
MARÍA ÁNGELES PASCUAL, SERGIO DÍAZ Y CELESTINO RODRÍGUEZ

EMPLEABILIDAD Y OCUPACIÓN LABORAL DE LOS EGRESADOS DEL MÁSTER EN FORMACIÓN

DEL PROFESORADO DE EDUCACIÓN SECUNDARIA OBLIGATORIA, BACHILLERATO Y FORMACIÓN...

\section{INTRODUCCIÓN}

La educación de posgrado a nivel de máster ha adquirido una importancia a nivel mundial. Tiene como finalidad la adquisición por el estudiante de una formación avanzada, de carácter especializado o multidisciplinar, orientado a la especialización académica o profesional, o bien a promover la iniciación en tareas investigadoras. Es sorprendente ver la variedad de tipos de programas de máster, además de la diversidad respecto a las características de los matriculados, desde recién licenciados que quieren complementar sus estudios a profesionales que desean formarse mejor. En la actualidad los títulos de postgrado suponen para muchos estudiantes un camino seguro que abrirá las puertas del mercado laboral (Sanchez, Pintado, Talledo y Carcelén, 2009).

En la Universidad española se pueden distinguir tres grandes grupos de estudios de posgrado (López, 2002):

- Programas de doctorado orientados a la innovación.

- Títulos propios, con una orientación más práctica y que se incluyen en la categoría de enseñanzas superiores no regladas, son estudios no homologados.

- Máster o Magister universitario. Son títulos a los que se accede desde un título de grado y tienen una duración que varía entre un año (60 créditos ECTS) a dos años (120 créditos ECTS).

Según los datos de Universia-España existen 4.678 títulos propios de posgrado, 3.600 másteres oficiales y 75 másteres oficiales Erasmus Mundus y se encuentran categorizados de la siguiente manera:

TABLA 1

Títulos de posgrado Universia-España, 2015

\begin{tabular}{|c|c|c|c|c|c|}
\hline \multicolumn{2}{|c|}{ Títulos PROPIOS } & \multicolumn{2}{c|}{ MÁSTERES OfICIALES } & \multicolumn{2}{c|}{ Másteres ERASMUS MUNDUS } \\
\hline Públicos & Privados & Públicos & Privados & Públicos & Privados \\
\hline 3.489 & 1.126 & 2.859 & 741 & 67 & 8 \\
\hline Presencial & A Distancia & Presencial & A Distancia & Presencial & A Distancia \\
\hline 2.108 & 709 & 3.109 & 148 & 62 & 1 \\
\hline On Line & Semipres. & On Line & Semipres. & On Line & Semipres. \\
\hline 518 & 348 & 187 & 306 & 4 & 3 \\
\hline & & Acreditados & No Acreditados & Acreditados & No Acreditados \\
\hline & & 2.744 & 856 & 18 & 61 \\
\hline
\end{tabular}

En la Universidad de Oviedo existen un total de 50 másteres oficiales que abarcan las cinco ramas de conocimiento y se encuentran adaptados al Espacio Europeo de Educación Superior. La duración de estos másteres oscila entre un año académico a tiempo completo (60 créditos ECTS) y 2 años (120 créditos ECTS). 
MARÍA ÁNGELES PASCUAL, SERGIO DÍAZ Y CELESTINO RODRÍGUEZ

EMPLEABILIDAD Y OCUPACIÓN LABORAL DE LOS EGRESADOS DEL MÁSTER EN FORMACIÓN

DEL PROFESORADO DE EDUCACIÓN SECUNDARIA OBLIGATORIA, BACHILLERATO Y FORMACIÓN...

Los estudios de máster desde 2007 tienen unas referencias legales (RD 1393/2007 y Orden ECI/3858/2007) y evaluativas (ANECA) para poder desarrollarse y posteriormente ser evaluados (verificación/seguimiento/acreditación). La ANECA y el Informe de Expertos encargado por el Ministerio de Educación, Cultura y Deporte instan a realizar estudios anuales para conocer la valoración y empleabilidad de los egresados y detectar los puntos débiles y fuertes del sistema universitario. Por otra parte, la European Association for Quality Assurance in Higher Education (2005) establece la necesidad de disponer de sistemas de información que permitan conocer la empleabilidad de los graduados, así como su valoración, como un indicador fundamental de la calidad de la formación impartida a través de la información de los propios egresados.

Como señalan Hernández, Tavera y Jimenez (2012: 42) «los egresados son el producto final que se obtiene después de transitar por un programa de posgrado", de ahí la importancia de conocer el camino que deciden tomar los egresados, su crecimiento y desempeño profesional son factores que deben ser analizados por la universidad y la administración educativa, para reflexionar sobre los resultados obtenidos de la formación realizada. Los estudios de egresados contribuyen a la construcción de un diagnóstico que sirve para estudiar las tendencias relacionadas con la ocupación, sueldo y tiempo que tardan en colocarse en el mercado laboral, satisfacción con la formación recibida y la aplicación de los conocimientos adquiridos en el empleo obtenido. Este tipo de estudios de egresados se ha implementado en las universidades en aras de introducir elementos de calidad en los sistemas de evaluación y como tal es contemplado en el Programa AUDIT de la ANECA para el Estado español.

Por otra parte, los estudios sobre empleabilidad de la población cualificada (EAE Business School, 2014) nos informan que una titulación superior reduce el riesgo de desempleo y permite acceder a mejores niveles salariales. Sin embargo, también observamos que la educación puede contribuir a una mayor empleabilidad solo si existen las oportunidades laborales para los trabajadores más productivos (Guzmán, Febles, Corredera, Flores, Tuyub y Rodríguez, 2008).

En el caso de la Universidad de Oviedo (UO) se ha habilitado desde la Unidad Técnica de Calidad el Procedimiento de gestión de la orientación profesional y seguimiento de egresados (PD-SGIC-UO-1.2.4.VO7). Llevar a cabo este procedimiento es cometido de las Comisiones de Calidad de los Centros de la UO, de las Comisiones de calidad por Máster Universitario y de las Comisiones de Calidad de cada Programa de Doctorado. Este procedimiento tiene para la UO varios objetivos:

1. Realizar un análisis de la información relativa al mercado laboral.

2. Definir las actuaciones en materia de orientación profesional.

3. Llevar un seguimiento de la gestión de esa orientación.

4. Revisar los informes de resultados de la encuesta de egresados.

5. Elaborar un informe de seguimiento que incluya las conclusiones y análisis de los resultados de las actuaciones de orientación profesional. 
MARÍA ÁNGELES PASCUAL, SERGIO DÍAZ Y CELESTINO RODRÍGUEZ

EMPLEABILIDAD Y OCUPACIÓN LABORAL DE LOS EGRESADOS DEL MÁSTER EN FORMACIÓN

DEL PROFESORADO DE EDUCACIÓN SECUNDARIA OBLIGATORIA, BACHILLERATO Y FORMACIÓN...

Este informe de conclusiones es posteriormente presentado a los Equipos Directivos de Centro y remitido al Vicerrectorado con competencias en materia de empleo y orientación profesional.

Sin embargo. y siguiendo a Ávila y Aguirre (2005), estos estudios deberían no solo investigar acerca del grado y tipo de inserción laboral de los egresados, sino también acerca de la valoración que hacen los egresados de las capacidades adquiridas, los perfiles profesionales reales, las funciones y tareas desempeñadas, las capacidades y perfiles demandados por los empleadores y la valoración que estos realizan de los conocimientos y capacidades de los egresados.

Un título de máster deberá garantizar, de acuerdo al Real Decreto 1393/2007, como mínimo las siguientes competencias básicas de los egresados:

- Que los estudiantes sepan aplicar los conocimientos adquiridos y su capacidad de resolución de problemas en entornos nuevos o poco conocidos dentro de contextos más amplios (o multidisciplinares) relacionados con su área de estudio;

- Que los estudiantes sean capaces de integrar conocimientos y enfrentarse a la complejidad de formular juicios a partir de una información que, siendo incompleta o limitada, incluya reflexiones sobre las responsabilidades sociales y éticas vinculadas a la aplicación de sus conocimientos y juicios;

- Que los estudiantes sepan comunicar sus conclusiones -y los conocimientos y razones últimas que las sustentan- a públicos especializados y no especializados de un modo claro y sin ambigüedades;

- Que los estudiantes posean las habilidades de aprendizaje que les permitan continuar estudiando de un modo que habrá de ser en gran medida autodirigido o autónomo.

El caso del Máster en Formación del Profesorado, uno de los contemplados en el RD 1393/2007, es un máster que habilita para el ejercicio de una actividad profesional regulada en España como es la docencia en Educación Secundaria $y$, por tanto, es el Gobierno quien establece las condiciones a las que debe de adecuarse el plan de estudios teniendo en cuenta la normativa europea. La Orden ECI/3858/2007 detalla el plan de estudios de manera que permita obtener las competencias necesarias para ejercer la profesión docente en la etapa educativa antes señalada. Las competencias que los estudiantes deben haber adquirido al concluir la titulación son:

1. Conocer los contenidos curriculares de las materias relativas a la especialización docente correspondiente, así como el cuerpo de conocimientos didácticos en torno a los procesos de enseñanza y aprendizaje respectivos. Para la formación profesional se incluirá el conocimiento de las respectivas profesiones.

2. Planificar, desarrollar y evaluar el proceso de enseñanza y aprendizaje potenciando procesos educativos que faciliten la adquisición de las competencias 
166 MARÍA ÁNGELES PASCUAL, SERGIO DÍAZ Y CELESTINO RODRÍGUEZ

EMPLEABILIDAD Y OCUPACIÓN LABORAL DE LOS EGRESADOS DEL MÁSTER EN FORMACIÓN

DEL PROFESORADO DE EDUCACIÓN SECUNDARIA OBLIGATORIA, BACHILLERATO Y FORMACIÓN...

propias de las respectivas enseñanzas, atendiendo al nivel y formación previa de los estudiantes, así como la orientación de los mismos, tanto individualmente como en colaboración con otros docentes y profesionales del centro.

3. Buscar, obtener, procesar y comunicar información (oral, impresa, audiovisual, digital o multimedia), transformarla en conocimiento y aplicarla en los procesos de enseñanza y aprendizaje en las materias propias de la especialización cursada.

4. Concretar el currículo que se vaya a implantar en un centro docente participando en la planificación colectiva del mismo; desarrollar y aplicar metodologías didácticas tanto grupales como personalizadas, adaptadas a la diversidad de los estudiantes.

5. Diseñar y desarrollar espacios de aprendizaje con especial atención a la equidad, la educación emocional y en valores, la igualdad de derechos y oportunidades entre hombres y mujeres, la formación ciudadana y el respeto de los derechos humanos que faciliten la vida en sociedad, la toma de decisiones y la construcción de un futuro sostenible.

6. Adquirir estrategias para estimular el esfuerzo del estudiante y promover su capacidad para aprender por sí mismo y con otros, y desarrollar habilidades de pensamiento y de decisión que faciliten la autonomía, la confianza e iniciativa personales.

7. Conocer los procesos de interacción y comunicación en el aula, dominar destrezas y habilidades sociales necesarias para fomentar el aprendizaje y la convivencia en el aula, y abordar problemas de disciplina y resolución de conflictos.

8. Diseñar y realizar actividades formales y no formales que contribuyan a hacer del centro un lugar de participación y cultura en el entorno donde esté ubicado; desarrollar las funciones de tutoría y de orientación de los estudiantes de manera colaborativa y coordinada; participar en la evaluación, investigación y la innovación de los procesos de enseñanza y aprendizaje.

9. Conocer la normativa y organización institucional del sistema educativo y modelos de mejora de la calidad con aplicación a los centros de enseñanza.

10. Conocer y analizar las características históricas de la profesión docente, su situación actual, perspectivas e interrelación con la realidad social de cada época.

11. Informar y asesorar a las familias acerca del proceso de enseñanza y aprendizaje y sobre la orientación personal, académica y profesional de sus hijos.

2. El máster en Formación del Profesorado de Educacion Secundaria Obligatoria, Bachillerato y Formación PROFESIONAL

Una de las principales salidas para los licenciados universitarios en los últimos años, y recientemente para las primeras promociones de graduados con el Espacio 
MARÍA ÁNGELES PASCUAL, SERGIO DÍAZ Y CELESTINO RODRÍGUEZ

EMPLEABILIDAD Y OCUPACIÓN LABORAL DE LOS EGRESADOS DEL MÁSTER EN FORMACIÓN

DEL PROFESORADO DE EDUCACIÓN SECUNDARIA OBLIGATORIA, BACHILLERATO Y FORMACIÓN...

Europeo de Educación Superior, ha sido el Máster en Formación del Profesorado de Secundaria. Muestra de ello son los datos que se reflejan en la Tabla 1, donde el máster supone entre un 15-18\% de los matriculados en máster en la Universidad de Oviedo y entre un 17-22\% de los egresados de máster de la universidad.

TABLA 2

Alumnos matriculados en título de Máster en la Universidad de Oviedo

\begin{tabular}{|l|c|c|c|c|c|c|c|}
\hline & \multicolumn{3}{|c|}{ MÁsTERES UNIVERSIDAD DE OVIEDO } & \multicolumn{3}{|c|}{ MÁSTER FORMACIÓN DEL PROFESORADO } \\
\cline { 2 - 8 } & $\begin{array}{c}\text { Plazas } \\
\text { ofertadas }\end{array}$ & Matriculados & Egresados & Matriculados & $\%$ & Egresados & $\%$ \\
\hline $2009-10$ & 455 & 835 & 649 & 191 & 18 & 185 & 22 \\
\hline $2010-11$ & 650 & 1.068 & 741 & 170 & 15 & 158 & 17 \\
\hline $2011-12$ & 992 & 939 & 704 & 187 & 16 & 168 & 19 \\
\hline $2012-13$ & 1.167 & 1.059 & 772 & 196 & 15 & 171 & 18 \\
\hline
\end{tabular}

Fuente: Vicerrectorado de Posgrado.

El Máster en Formación del Profesorado de Educación Secundaria se caracteriza, en primer lugar, por su transversalidad, ya que ofrece una especialidad para cada uno de los campos del saber, correspondiente a las diferentes especialidades de profesores del cuerpo 0590, de Secundaria, aunque también otros cuerpos como los de profesores de Formación Profesional y de Escuela Oficial de Idiomas. Sin embargo, y desafortunadamente, la coyuntura económica ha conllevado una reducción de la tasa de reposición de todos los cuerpos de funcionarios públicos estableciéndose primero en un 30\% hasta 2011, y tras los presupuestos de 2012 en un $10 \%$.

Ante estas condiciones, las previsiones de empleo en el sector público y también en el concertado y privado son poco halagüeñas. Estas expectativas negativas se unen a un sustrato que hace bastante difícil la incorporación de profesorado a la red pública y concertada asturiana, la baja natalidad en las dos últimas décadas y la emigración juvenil por razones laborales a otras regiones de España. Este es precisamente el caso asturiano que vamos a estudiar en detalle a través de este artículo, centrándonos en el devenir de los egresados del Máster en Formación del Profesorado de Educación Secundaria, Bachillerato y Formación Profesional, impartido en la Facultad de Formación del Profesorado y Educación de la Universidad de Oviedo desde el curso académico 2009-2010, y tomando como población sus cuatro primeras promociones.

El Máster en Formación del Profesorado en Educación Secundaria de la Universidad de Oviedo ha convocado en los últimos cuatro años entre 190 y 200 plazas, excepto el primer año que se convocaron 240 plazas, pero solo fueron cubiertas 200 plazas.

Presentamos a continuación algunos datos que definen el máster en nuestra universidad y que tienen relevancia para la interpretación de los resultados de la encuesta. 
MARÍA ÁNGELES PASCUAL, SERGIO DÍAZ Y CELESTINO RODRÍGUEZ

EMPLEABILIDAD Y OCUPACIÓN LABORAL DE LOS EGRESADOS DEL MÁSTER EN FORMACIÓN

DEL PROFESORADO DE EDUCACIÓN SECUNDARIA OBLIGATORIA, BACHILLERATO Y FORMACIÓN...

El número de plazas se distribuye de forma heterogénea entre 17 especialidades durante los cursos 2009 a 2013 (en el curso 2013-14 se incorpora una especialidad más).

Son las especialidades de Geografía e Historia, Lengua Castellana y Literatura, Orientación educativa e inglés las que más alumnos demandan, mientras que las especialidades de Asturiano y Lenguas clásicas son las que menos egresados tienen. Esta distribución fue pactada inicialmente entre la Universidad y la Consejería de Educación en función del mercado de empleabilidad de la Comunidad, pero posteriormente se fueron adaptando a las demandas existentes entre los egresados de licenciatura y grado que solicitaban el máster. En el Principado de Asturias, desde la puesta en marcha del máster, solo se han convocado plazas de empleo público para Educación Secundaria en dos ocasiones, una convocatoria en el año 2010 a la que pudieron presentarse los egresados del máster de ese mismo curso y otra convocatoria en el año 2014. Teniendo en cuenta que los egresados del máster se pueden presentar en otras CC. AA., consideramos de interés presentar la oferta de plazas públicas en las diferentes CC. AA. en estos cuatro años.

TABLA 3

Plazas de empleo público en Educación Secundaria ofertadas en las diferentes convocatorias en todo el territorio español

\begin{tabular}{|c|c|c|c|c|}
\hline & $\begin{array}{c}\text { CONVOCATORIA } \\
2010 \\
\end{array}$ & $\begin{array}{c}\text { CONVOCATORIA } \\
2011 \\
\end{array}$ & $\begin{array}{c}\text { CONVOCATORIA } \\
2012 \\
\end{array}$ & $\begin{array}{c}\text { CONVOCATORIA } \\
2013\end{array}$ \\
\hline Andalucía & 3.141 & 0 & 1.922 & 0 \\
\hline Aragón & 340 & 0 & 0 & 0 \\
\hline Asturias & 108 & 0 & 0 & 0 \\
\hline Baleares & 327 & 0 & 0 & 0 \\
\hline Canarias & 505 & 0 & 0 & 0 \\
\hline Castilla-La Mancha & 580 & 0 & 0 & 0 \\
\hline Castilla-León & 439 & 0 & 0 & 0 \\
\hline Extremadura & 297 & 0 & 0 & 0 \\
\hline Galicia & 272 & 0 & 0 & 0 \\
\hline La Rioja & 61 & 0 & 0 & 0 \\
\hline Madrid & 1.100 & 0 & 190 & 0 \\
\hline Murcia & 481 & 0 & 0 & 0 \\
\hline Navarra & 68 & 0 & 0 & 0 \\
\hline Valencia & 475 & 0 & 0 & 30 \\
\hline Ceuta y Melilla & 10 & 0 & 0 & 0 \\
\hline Cataluña & 0 & 1.005 & 0 & 0 \\
\hline Barcelona & 0 & 34 & 0 & 0 \\
\hline País Vasco & 0 & 225 & 328 & 0 \\
\hline Cantabria & 0 & 0 & 25 & 0 \\
\hline Total & 8.204 & 1.264 & 2.465 & 30 \\
\hline
\end{tabular}


MARÍA ÁNGELES PASCUAL, SERGIO DÍAZ Y CELESTINO RODRÍGUEZ

EMPLEABILIDAD Y OCUPACIÓN LABORAL DE LOS EGRESADOS DEL MÁSTER EN FORMACIÓN

DEL PROFESORADO DE EDUCACIÓN SECUNDARIA OBLIGATORIA, BACHILLERATO Y FORMACIÓN...

\section{Metodología}

La presente investigación es de tipo exploratorio y se presenta como el primer estudio de egresados del Máster en Formación del Profesorado de la Universidad de Oviedo. Hay que señalar que la opinión de los egresados en torno al ámbito laboral es una fuente básica de información para la toma de decisiones sobre el máster, que permite fortalecer el funcionamiento del mismo.

Hemos planteado el análisis desde el estudio de casos al circunscribirnos a los alumnos egresados del Máster en Formación del Profesorado de Educación Secundaria Obligatoria, Bachillerato y Formación Profesional de la Facultad de Formación del Profesorado y Educación de la Universidad de Oviedo.

Los objetivos planteados en el estudio son:

- Conocer la relación entre la formación que se proporciona en el máster y el desempeño profesional.

- Conocer la empleabilidad de los egresados del máster.

- Conocer la aplicación de las competencias adquiridas en el máster.

Para dar respuesta a estos objetivos se llevó a cabo una revisión exhaustiva de la literatura existente con el fin de identificar las mejores prácticas. Después de la cual se llegó a la conclusión de que los instrumentos más empleados en la recogida de información eran la encuesta y la entrevista. Consideramos que la encuesta telefónica nos permitiría llegar mejor a los egresados y llevamos a cabo el diseño de la misma elaborada "ad hoc» (EnEgMfp) y aplicada de forma directa a los egresados. Este procedimiento nos permitió llegar a un mayor número de activos que se encontraban dispersos por la geografía nacional. Para ello se recurrió a un entrevistador formado, bajo supervisión y control del trabajo y conocedor de los objetivos del estudio.

La población objeto de estudio es de 682 alumnos pertenecientes a las cuatro primeras promociones de egresados del Máster en Formación del Profesorado de la Universidad de Oviedo, concretamente de los cursos 2009/2010, 2010/2011, 2011/2012 y 2012/2013. El número de egresados con que cuenta este máster es del $91,6 \%$, lo cual define la eficiencia terminal del mismo.

TABLA 4

Población objeto de estudio de las cuatro promociones

\begin{tabular}{|l|c|c|c|}
\hline & MATRICULADOS & EGRESADOS & CONTESTAN A LA ENCUESTA \\
\hline $2009-2010$ & 191 & 185 & 71 \\
\hline $2010-2011$ & 170 & 158 & 67 \\
\hline $2011-2012$ & 187 & 168 & 62 \\
\hline $2012-2013$ & 196 & 171 & 74 \\
\hline TOTAL & 744 & 682 & 274 \\
\hline
\end{tabular}


MARÍA ÁNGELES PASCUAL, SERGIO DÍAZ Y CELESTINO RODRÍGUEZ

EMPLEABILIDAD Y OCUPACIÓN LABORAL DE LOS EGRESADOS DEL MÁSTER EN FORMACIÓN

DEL PROFESORADO DE EDUCACIÓN SECUNDARIA OBLIGATORIA, BACHILLERATO Y FORMACIÓN...

Para la administración del cuestionario se contactó con todas las personas objeto del estudio con la finalidad de averiguar si deseaban participar en la investigación. Los datos obtenidos han sido los correspondientes a 274 egresados que han contestado la encuesta, lo cual representa un 34\% de la población, que podemos considerar representativa de los egresados del máster.

\subsection{Descripción de la encuesta}

El diseño del instrumento se realizó según los objetivos formulados. Las variables incluidas en la encuesta son las siguientes:

1. Rasgos generales del egresado. Aquí se captan los datos personales de los egresados, nombre, edad, sexo, título de acceso al máster y año en que realizó el máster. Estos componentes tienen un peso específico en cuestiones objetivas como es el ingreso al mercado laboral.

2. Ubicación en el mercado de trabajo. El análisis de estos datos complementa la relación entre educación y mercado de trabajo. Integra dos tipos de preguntas marcadas por la situación de empleo o desempleo. En la situación de empleo se pregunta por los datos del empleo, régimen jurídico de la empresa o institución, tipo de contrato y tiempo que tardó en encontrar trabajo. En la situación de desempleo se pregunta por su historia laboral desde la obtención del título del máster, tiempo que lleva desempleado, así como la asistencia de un orientador laboral y las expectativas laborales. Lo que nos proponemos es conocer a qué se dedican los egresados del máster con trabajo, la relación con el máster que han estudiado, las condiciones de sus contratos y si la red pública o la privada-concertada se nutren de estos profesionales. Y, por otra parte, conocer si los egresados desempleados han tenido la oportunidad de aplicar en el mundo laboral las competencias adquiridas en el máster y hasta qué punto ha sido activa su búsqueda de empleo y qué herramientas han utilizado.

3. Formación adquirida con posterioridad. En este apartado se trató de detectar si continúa formándose en el ámbito para el cual se diseñó el máster, es decir, para acceder a una plaza como profesor de educación secundaria y qué tipo de formación realizan.

\section{ANÁLISIS DE LOS DATOS OBTENIDOS}

Hemos podido comprobar que el perfil de los alumnos que han cursado el máster ha variado ligeramente durante los últimos años. No tanto en cuanto al sexo, que es de 7 mujeres cada 3 hombres, pero sí en cuanto a la edad, con un rejuvenecimiento del alumnado. Tal y como puede observarse en la Figura 2 la media de edad decrece promoción tras promoción, de 33 a 29 años, y una edad mínima que va disminuyendo también de 25 a 23 años. 
MARÍA ÁNGELES PASCUAL, SERGIO DÍAZ Y CELESTINO RODRÍGUEZ

EMPLEABILIDAD Y OCUPACIÓN LABORAL DE LOS EGRESADOS DEL MÁSTER EN FORMACIÓN DEL PROFESORADO DE EDUCACIÓN SECUNDARIA OBLIGATORIA, BACHILLERATO Y FORMACIÓN...

\section{FIGURA 1}

Distribución por sexos del alumnado del máster en las cuatro promociones

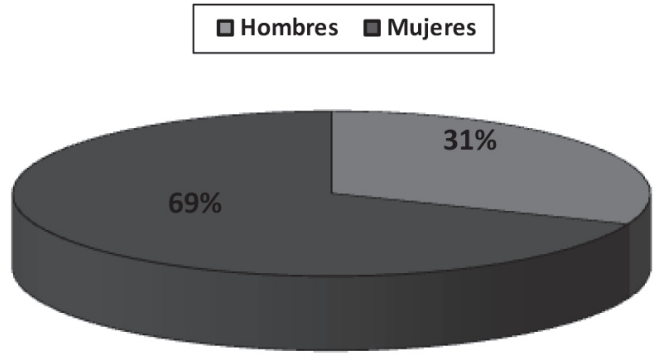

FIGURA 2

Edad media, máxima y mínima en las cuatro promociones

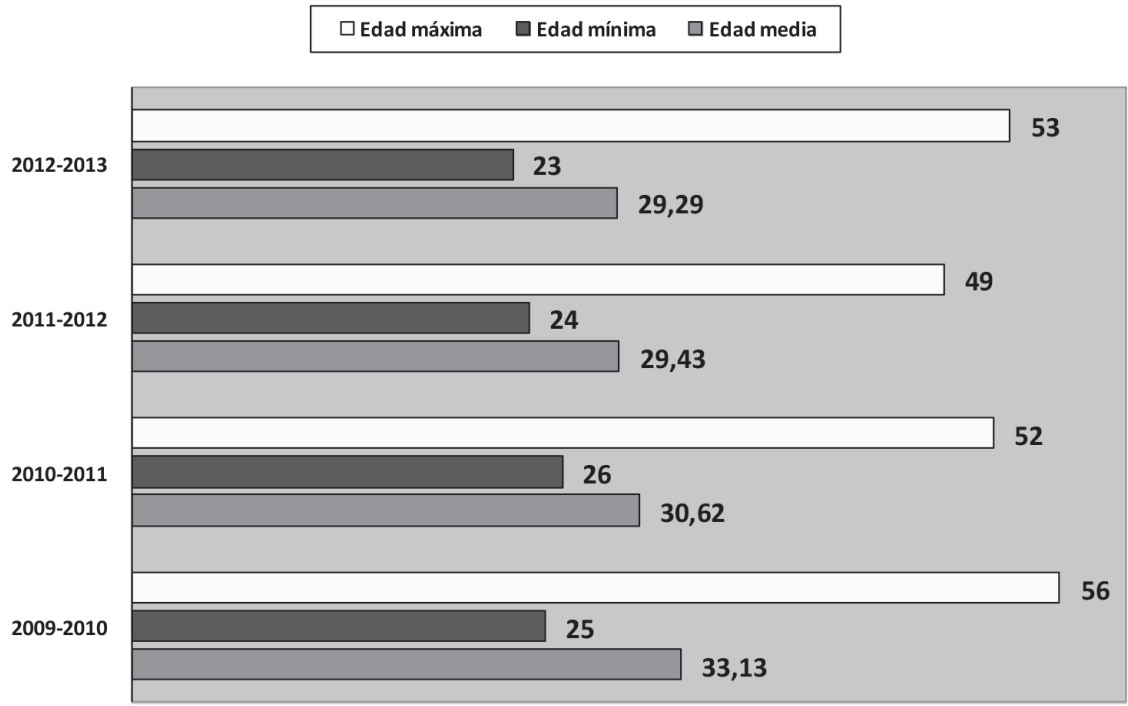

Para comenzar el análisis de los datos obtenidos, cabe decir que el 52\% de los egresados del máster manifiesta estar trabajando, lo cual abre perspectivas positivas con arreglo a las competencias con las que el máster provee a su alumnado. Sin embargo, habría que preguntarse si las competencias adquiridas guardan relación con el empleo. Lo revelador es que el 58,27\% de los encuestados no están trabajando en cuestiones relacionadas con la formación adquirida en el máster. Y un $41 \%$ de los empleados afirma tener un puesto de trabajo relacionado con las competencias adquiridas en el máster 
MARÍA ÁNGELES PASCUAL, SERGIO DÍAZ Y CELESTINO RODRÍGUEZ

EMPLEABILIDAD Y OCUPACIÓN LABORAL DE LOS EGRESADOS DEL MÁSTER EN FORMACIÓN

DEL PROFESORADO DE EDUCACIÓN SECUNDARIA OBLIGATORIA, BACHILLERATO Y FORMACIÓN...

Como muestra la Figura 3 la mayor parte de los trabajos no son ni como autónomos ni como docentes en centros públicos, privados o concertados, sino un apartado encabezado por un término paraguas, "Otros». Aquí los egresados mencionan como ocupaciones principales las de administrativo, vendedor y, con bastante frecuencia, los centros privados de formación no reglada; en otras palabras, dar clases en academias. Esto ocurre con el 45\% de los egresados que están trabajando en estos momentos.

Un 20\% de los encuestados que se encuentra en situación de empleo lo hace por cuenta ajena en un centro privado/concertado y un $21 \%$ lo hacen por cuenta ajena en un centro público.

FIGURA 3

Naturaleza de las ocupaciones de los egresados del máster
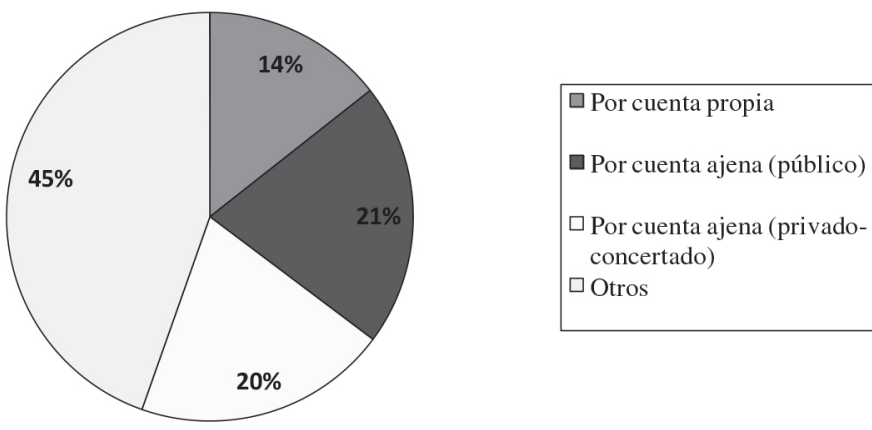

En casos minoritarios los egresados trabajan con plaza fija o como interinos a tiempo completo o parcial como profesores de Secundaria o conservatorio en la red pública. Este trabajo también se menciona en alguna ocasión en la red privada y concertada, aunque más bien como profesores de actividades extraescolares que se realizan en los centros. El grupo menos numeroso, un 14,39\%, es el de los trabajadores por cuenta propia, si bien aportan mayor estabilidad y jornadas que tienden más a ser completas.

En cuanto a la calidad de estos empleos, hay que destacar que la mayoría son temporales (58\%), aunque la precariedad asociada a la incertidumbre se ve atenuada por una mayor incidencia de las jornadas completas (57\%) frente a las parciales o los contratos por horas. En el caso de los egresados con trabajo, la inmensa mayoría (89\%) lo ha encontrado antes de seis meses después de titular, mientras que en porcentajes similares al 10\% lo han hecho en los intervalos siguientes, como muestra la figura 4. 
MARÍA ÁNGELES PASCUAL, SERGIO DÍAZ Y CELESTINO RODRÍGUEZ

EMPLEABILIDAD Y OCUPACIÓN LABORAL DE LOS EGRESADOS DEL MÁSTER EN FORMACIÓN DEL PROFESORADO DE EDUCACIÓN SECUNDARIA OBLIGATORIA, BACHILLERATO Y FORMACIÓN...

TABLA 5

Tipos de contrato de los egresados con empleo

\begin{tabular}{|l|c|}
\hline \multicolumn{1}{|c|}{ TIPO DE CONTRATO } & PROPORCIÓN DE EGRESADOS CON EMPLEO \\
\hline Temporal & $58 \%$ \\
\hline Indefinido & $42 \%$ \\
\hline A media jornada & $43 \%$ \\
\hline Jornada completa & $57 \%$ \\
\hline
\end{tabular}

No se perciben diferencias en lo concerniente a la empleabilidad entre las diferentes promociones, si bien parece haberlas según la titulación. Así en la especialidad de inglés parece que muchos egresados han conseguido ganarse la vida, al igual que los provenientes de carreras de ciencias, quizás por poder combinar la profesión docente con otras salidas.

En una proporción mucho menor a las de los egresados que están en activo, con un $25,82 \%$, los hay también que se encuentran en situación de desempleo. De estos, un $36,23 \%$ no ha disfrutado nunca de contratos relacionados con la labor docente para la que habilita el máster, mientras que el resto, mayoritario, sí. Se da la paradoja de que la mayoría de los egresados desempleados lleva en desempleo o bien muy poco tiempo o bien mucho (más de dos años).

\title{
FIGURA 4
}

Tiempo de desempleo de los egresados en paro

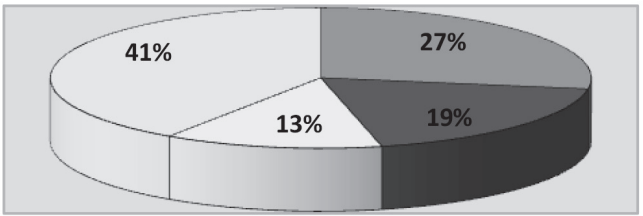

\author{
$\square$ Menos de 6 meses $(27,54 \%)$ \\ 口Entre 6 meses y 1 año $(18,84 \%)$ \\ Entre 1 y 2 años $(13,04 \%)$ \\ $\square$ Más de 2 años $(40,58 \%)$
}

Un $62,32 \%$ de los egresados que se encuentran sin empleo ha probado las fórmulas tradicionales para buscarlo. Sin embargo, un 37,68\% afirma haber recurrido a un orientador laboral. Este porcentaje aumenta a medida que avanzamos en el tiempo, con unos egresados de menor edad y que han finalizado sus estudios en años académicos menos lejanos. La tabla siguiente muestra cómo el porcentaje de egresados que recurre a orientadores laborales crece en este sentido, aunque también hay muchos egresados de la primera promoción que han hecho uso. 
MARÍA ÁNGELES PASCUAL, SERGIO DÍAZ Y CELESTINO RODRÍGUEZ

EMPLEABILIDAD Y OCUPACIÓN LABORAL DE LOS EGRESADOS DEL MÁSTER EN FORMACIÓN DEL PROFESORADO DE EDUCACIÓN SECUNDARIA OBLIGATORIA, BACHILLERATO Y FORMACIÓN...

TABLA 6

Egresados asesorados por un orientador laboral

\begin{tabular}{|c|c|}
\hline PROMOCIÓN-CURSO & EGRESADOS ASESORADOS POR ORIENTADOR LABORAL \\
\hline $2009-2010$ & $55 \%$ \\
\hline $2010-2011$ & $20 \%$ \\
\hline $2011-2012$ & $26,7 \%$ \\
\hline $2012-2013$ & $50 \%$ \\
\hline
\end{tabular}

A pesar de las estadísticas, el 53,62\% se muestra optimista y piensa encontrar un empleo relacionado con el máster en el futuro, frente a un 46,38\% desesperanzado. Tanto si se encuentran en una situación activa, como en situación de desempleo, gran número de egresados del máster, el 31,08\%, continúa formándose, en el primer caso para obtener una plaza en convocatorias de empleo público, sobre todo aquellas para las que habilita el máster, o mejorar su situación laboral; y en el segundo, para salir de la situación de paro con esperanzas de conseguir un empleo como docentes. Dentro de esta formación, un 75,9\% la orienta hacia esferas relacionadas con el máster, mientras que un 21,4\% sigue formaciones regladas o sistematizadas suficientemente, aunque sin tanta relación, y un 2,4\% otro tipo de formación que no detallan.

Preguntados acerca del tipo de formación que siguen, el 60,24\% de los egresados, es decir, la amplísima mayoría, se prepara para las oposiciones a profesor de Secundaria, y un porcentaje también significativo, el 14,46\%, relacionada con el máster aunque no necesariamente para la realización de oposiciones, como magisterio o cursos que podrían puntuar como méritos según el baremo de algunas comunidades autónomas. Un 18,07\% de los encuestados dicen haber vuelto a la formación básica que les aportaba su licenciatura, y finalmente un 0,02\% prepara oposiciones a cuerpos de funcionarios diferentes a los docentes. Algo importante a destacar es cómo, a medida que avanzamos en las promociones, cada vez hay más egresados que siguen una formación. Esto puede deberse, fundamentalmente, a que son más jóvenes y a que han acabado sus estudios en un momento muy complicado para hacerse un hueco en el mercado laboral europeo.

\section{CONClusiones}

El seguimiento de egresados es una poderosa herramienta que permite analizar la situación que viven los egresados en su vida laboral y la forma en que esta impacta directamente en su bienestar económico. El esfuerzo y la dedicación de concluir un posgrado ha de representar la apertura a mejores oportunidades que conlleven el crecimiento laboral. La investigación ha permitido conocer la empleabilidad de los alumnos del máster y el elevado porcentaje de egresados que han obtenido el grado académico. 
Podemos decir que la tasa de empleabilidad de los egresados del Máster en Formación del Profesorado se aleja considerablemente de los datos de las tasas de actividad de la Encuesta de Población Activa a nivel nacional que se encuentra en el 89\% para una formación de Educación superior en la franja de edad de 25-29 años y se incrementa en la franja de 30 a 34 años al 92,7\%. Sin embargo, en la Comunidad Autónoma del Principado de Asturias la situación es bien distinta ya que las tasas de actividad con titulación superior no superan el 45\%. Sin embargo, y según el estudio de egresados de máster de la UO, un 71,6\% se encuentra trabajando después de concluir sus estudios, mientras que sólo el 53,6\% de los egresados del Máster en Formación del Profesorado afirma encontrar un empleo, encontrándose por tanto por encima de la media de la población asturiana con estudios superiores, pero por debajo del resto de egresados de máster.

El Máster en Formación del Profesorado mantiene depositadas en sí las expectativas y sueños de trabajo docente de muchos jóvenes que finalizan sus licenciaturas y estudios de grado hoy en día. Prueba de ello es que año tras año se cubren las plazas ofertadas, quedando gente incluso en las listas de espera, y el número de alumnos que lo finaliza es también mayor. No obstante, la mitad aproximadamente de los egresados de las cuatro primeras promociones tiene un puesto de trabajo. Sin embargo, esto no debe llevarnos a un triunfalismo exacerbado, ya que las condiciones no son buenas en todos los casos.

En primer lugar, gran parte de estos empleos son temporales, con la carga de precariedad que ello implica y, aunque la mayor parte sean a jornada completa, no deja de haber contratos a tiempo parcial y por horas que impiden la emancipación correcta de los egresados y su inserción completa en el mundo laboral. La situación de estos años ha hecho que no se convocaran oposiciones en nuestra comunidad autónoma, y que, en aquellas donde se convocan, el número de plazas ha sido muy bajo. En tanto en cuanto estas restricciones no se moderen, los egresados del máster van a adolecer de falta de trabajo como profesionales de la enseñanza en plenitud, que les permita desarrollar sus habilidades.

Es cuando menos llamativo, sin embargo, que otro sector de egresados se haya estancado tanto, sin importar la fecha en la que hayan terminado el máster ni el género ni la edad ni la especialidad. De los egresados desempleados, la mayoría nunca han trabajado como docentes después del máster. Se abre una dicotomía muy clara entre egresados que llevan mucho tiempo en paro y egresados que trabajan o llevan poco tiempo sin empleo; entre aquellos que sólo buscan empleo por las vías tradicionales y aquellos que recurren a profesionales, y entre los que conservan la esperanza de un futuro como profesores y los que ya lo descartan. El desempleo es menor entre los titulados procedentes de carreras técnicas, como arquitectura o ingeniería, y también en algunas de ciencias sociales como derecho, ya que son estudios muy eclécticos que permiten trabajar en campos muy diversos.

En relación con el desempleo, huelga decir que los que menos tiempo han estado buscando empleo son los que más se han animado a recurrir a la orientación laboral de un profesional. De ahí quizás un mayor éxito a la hora de encontrar 
MARÍA ÁNGELES PASCUAL, SERGIO DÍAZ Y CELESTINO RODRÍGUEZ

EMPLEABILIDAD Y OCUPACIÓN LABORAL DE LOS EGRESADOS DEL MÁSTER EN FORMACIÓN

DEL PROFESORADO DE EDUCACIÓN SECUNDARIA OBLIGATORIA, BACHILLERATO Y FORMACIÓN...

trabajo. Si lo ponemos en relación con los datos acerca de los egresados con empleo, podemos establecer una relación de proporcionalidad directa entre la utilización de este servicio con las mayores probabilidades de encontrar un empleo (a muchos egresados les costaba mucho tiempo encontrar un empleo, mientras que otros lo habían encontrado en apenas seis meses).

Parece que los egresados no tiran la toalla en lo que se refiere a buscar la posibilidad de un empleo digno y estable como docentes. Gran parte de ellos, estén trabajando o no, continúan formándose ya sea en líneas paralelas para tener más puertas abiertas como, mayoritariamente, en la preparación de oposiciones a profesores de Secundaria, conservatorio, EOI, etc. De hecho, en muchos casos no sería erróneo pensar que la formación que siguen tras el máster (segunda licenciatura o segundo grado, doctorado, másteres, ciclos formativos...) podrá ser alegada por los egresados como mérito en futuros procesos selectivos docentes. A medida que vamos avanzado en promociones del máster, aumenta el número de egresados preparándose, fruto de una menor edad y de la coyuntura socioeconómica actual, que les impide encontrar el empleo que buscan o, simplemente, un puesto de trabajo.

Por otra parte, consideramos que para continuar con el seguimiento de los egresados es importante tener una base de datos actualizada que permita tener contacto con ellos y con sus empleadores.

\section{REFERENCIAS BIBLIOGRÁFICAS}

Ávila, M. y Aguirre, C. (2005). El seguimiento de egresados como indicador de la calidad docente. Revista Electrónica Interuniversitaria de Formación del Profesorado, 8 (3). Descargado el 15 de setiembre de 2014. http://redalyc.uaemex.mx/redalyc/src/inicio/ ArtPdfRed.jsp?iCve=217017156006.

European Association form Quality Assurance in Higher Education (2005). Criterios y directrices para la garantía de Calidad en el Espacio Europeo de Educación Superior. http:// www.enqa.net/bologna.lasso. Traducción ANECA.

Guzmán, S.; Febles, M.; Corredera, A.; Flores, P.; Tuyub, A. y Rodríguez, P. A. (2008). Estudio de seguimiento de egresados: recomendaciones para su desarrollo. Innovación Educativa, vol. 8 (42), 19-31.

Hernández, C. A.; Tavera, M. E. y Jiménez, M. (2012). Seguimiento de egresados en tres programas de maestría en una escuela del Instituto Politécnico Nacional de México. Formación Universitaria, 5 (2), 41-52.

Instituto Nacional de Estadística (2013). Encuesta de población activa. Descargado el 26 de setiembre de 2014. http://www.ine.es/daco/daco42/daco4211/epa0313.pdf.

León, G. y Montero, I. (2002). Métodos de investigación en psicología y educación. Madrid: McGrawHill.

López, M. A. (2002). Los estudios de postgrado en el mundo. Revista de Enseñanza Universitaria, 20, 65-74.

Orden ECI/3858/2007 por la que se establecen los requisitos para la verificación de los títulos universitarios oficiales que habiliten para el ejercicio de las profesiones de Profesor de Educación Secundaria Obligatoria y Bachillerato, Formación Profesional y Enseñanza de Idiomas. Boletín Oficial del Estado, 29 de diciembre de 2007, 53751-53753. 
MARÍA ÁNGELES PASCUAL, SERGIO DÍAZ Y CELESTINO RODRÍGUEZ

EMPLEABILIDAD Y OCUPACIÓN LABORAL DE LOS EGRESADOS DEL MÁSTER EN FORMACIÓN DEL PROFESORADO DE EDUCACIÓN SECUNDARIA OBLIGATORIA, BACHILLERATO Y FORMACIÓN...

Resolución de 23 de abril de 2010 por la que se convocan procedimientos selectivos para el acceso e ingreso en los cuerpos docentes. Boletín Oficial del Principado de Asturias.

Real Decreto 1393/2007 por el que se establece la ordenación de las enseñanzas universitarias oficiales. Boletín Oficial del Estado, 30 de octubre de 2007, 44037-44048.

Ruiz, J. I. (1999). Metodología de la investigación cualitativa. Bilbao: Universidad de Deusto.

Sánchez, J.; Pintado, T.; Talledo, H. y Carcelén, S. (2009). La educación de posgrado en España. Un estudio empírico de la estructura de motivaciones latentes. Innovar, Especial en Educación, dic. 2009, 131-140.

Sevillano, M. L.; Quicios, P. y González, J. L. (2016). Posibilidades ubicuas del ordenador portátil: percepción de estudiantes universitarios españoles. Comunicar, 46, 87-95. Doi http://dx.doi.org/10.3916/C47-2016-09.

Unidad Técnica de Calidad de la Universidad de Oviedo. Procedimiento de gestión de la orientación profesional y seguimiento de egresados (PD-SGIC-UO-1.2.4.vO7), 1-6. Descargado el día 25 de setiembre de 2014 file://C:/Users/Angeles/Downloads/1.2.4_Orientacion_profesional_y_Registros\%20(2).pdf. 
